\title{
Engineering covalently bonded 2D layered materials by self-intercalation
}

Zhao, Xiaoxu; Song, Peng; Wang, Chengcai; Riis-Jensen, Anders Christian; Fu, Wei; Deng, Ya; Wan, Dongyang; Kang, Lixing; Ning, Shoucong; Dan, Jiadong

Total number of authors:

17

Published in:

Nature

Link to article, DOI:

$10.1038 / \mathrm{s} 41586-020-2241-9$

Publication date:

2020

Document Version

Peer reviewed version

Link back to DTU Orbit

Citation (APA):

Zhao, X., Song, P., Wang, C., Riis-Jensen, A. C., Fu, W., Deng, Y., Wan, D., Kang, L., Ning, S., Dan, J., Venkatesan, T., Liu, Z., Zhou, W., Thygesen, K. S., Luo, X., Pennycook, S. J., \& Loh, K. P. (2020). Engineering covalently bonded 2D layered materials by self-intercalation. Nature, 581(7807), 171-177.

https://doi.org/10.1038/s41586-020-2241-9

\section{General rights}

Copyright and moral rights for the publications made accessible in the public portal are retained by the authors and/or other copyright owners and it is a condition of accessing publications that users recognise and abide by the legal requirements associated with these rights.

- Users may download and print one copy of any publication from the public portal for the purpose of private study or research.

- You may not further distribute the material or use it for any profit-making activity or commercial gain

- You may freely distribute the URL identifying the publication in the public portal 


\section{Engineering Covalently Bonded 2D Layered Materials by Self- 2 Intercalation}

3 Xiaoxu Zhao ${ }^{1,2}$, Peng Song ${ }^{2}$, Chengcai Wang ${ }^{3}$, Anders C. Riis-Jensen ${ }^{4}$, Wei Fu², Ya Deng ${ }^{5}$, Dongyang Wan ${ }^{6}$, 4 Lixing Kang ${ }^{5}$, Shoucong Ning ${ }^{1}$, Jiadong Dan ${ }^{1}$, T. Venkatesan ${ }^{1,6}$, Zheng Liu ${ }^{5}$, Wu Zhou ${ }^{7}$, Kristian S. Thygesen $^{4}$,

5 Xin Luo ${ }^{8^{*}}$, Stephen J. Pennycook ${ }^{1^{*}}$, and Kian Ping Loh ${ }^{2 *}$

$6{ }^{1}$ Department of Materials Science and Engineering, National University of Singapore, 9 Engineering Drive $7 \quad 1,117575$, Singapore

8 2Department of Chemistry and Centre for Advanced 2D Materials, National University of Singapore, 3

9 Science Drive 3, Singapore, 117543, Singapore

$10{ }^{3}$ Department of Electrical and Electronic Engineering, Southern University of Science and Technology,

11 Shenzhen, 518055, China

$12{ }^{4}$ CAMD and Center for Nanostructured Graphene (CNG), Department of Physics, Technical University of 13 Denmark, DK-2800 Kongens Lyngby, Denmark

$14{ }^{5}$ School of Materials Science and Engineering, Nanyang Technological University, Singapore, 639798, 15 Singapore

$16{ }^{6}$ NUSNNI-NanoCore, National University of Singapore, Singapore 117411

$17{ }^{7}$ School of Physical Sciences and CAS Centre for Excellence in Topological Quantum Computation, 18 University of Chinese Academy of Sciences, Beijing 100049, China

$19{ }^{8}$ State Key Laboratory of Optoelectronic Materials and Technologies, Centre of Physical Mechanics and Biophysics, Sun Yat-sen University, Guangzhou 510275, China

21 Correspondence and requests for materials should be addressed to X. L. (luox77@mail.sysu.edu.cn) S. J.

22 P (steve.pennycook@nus.edu.sg) \& K. P. L ( $\underline{\text { chmlohkp@nus.edu.sg) }}$

23 X. Zhao \& P. Song contributed equally to this work

Abstract:

Two-dimensional (2D) materials ${ }^{1-5}$ offer a unique platform for exploring the physics of topology and many-body phenomena. Filling the van der Waals (vdW) gap of 2D materials with intercalants is expected to create new properties ${ }^{6,7}$, but post-growth intercalation is usually limited to alkali metals ${ }^{8-}$ 
dichalcogenides (TMDs) during growth creates a whole new class of ultrathin, covalently bonded materials (named ic-2d). The stoichiometry of ic-2d is defined by periodic occupancy patterns of the octahedral vacancy sites in the vdW gap, and its properties can be tuned by varying the coverage and spatial arrangement of the filled sites ${ }^{7,13}$. By performing growth under conditions of high metal chemical potential ${ }^{14,15}$, we can grow a full spectrum of Ta-intercalated TaS(Se) $)_{y}$ including $25 \%$ intercalated Tag $\mathrm{S}_{16}$, 33.3\% intercalated $\mathrm{Ta}_{7} \mathrm{~S}_{12}, 50 \% \mathrm{Ta}_{10} \mathrm{~S}_{16}, 66.7 \% \mathrm{Ta}_{8} \mathrm{Se}_{12}$ (Kagome lattice) and 100\% $\mathrm{Ta}_{9} \mathrm{Se}_{12}$. Ferromagnetic order was detected for some of the intercalated phases. We have also verified that self-intercalated intercalation as a novel approach to grow a new class of ic-2d materials with stoichiometry or composition-dependent properties.

Introduction

The surge in 2D materials research has heralded a new branch of condensed-matter physics concerned with the description of electrons in atomically thin structures. Thus far, research efforts have primarily focused on 2D monolayers ${ }^{2}$ and their hetero-stacked structures $^{3}$, in which new properties can be engineered by generating superlattices of different Moiré wavelengths. However, these hetero-stacked structures are currently produced by bottom-up methods that have low yield and poor reproducibility ${ }^{16}$. An alternative way of compositional tuning is based on the intercalation of foreign atoms in the vdW gap sandwiched by the chalcogen atoms; this has been shown to induce pseudo-2D characteristics in bulk crystals and modify their electronic properties ${ }^{4,6,7}$. Depending on the interlayer stacking registries, the vdW gaps in TMDs contain octahedral and tetrahedral vacancies or trigonal-prismatic vacancies ${ }^{13}$, which provide docking sites for a diverse range of intercalants. Examples of successful intercalants include alkali

51 metals ${ }^{8-10}$ such as $\mathrm{Li}, \mathrm{Na}, \mathrm{K}$; transition metals ${ }^{17-21}$ such as $\mathrm{Cu}, \mathrm{Co}, \mathrm{Ni}, \mathrm{Fe}, \mathrm{Nb}, \mathrm{Sn}$; noble metals ${ }^{22-24}$ such as $52 \mathrm{Ag}, \mathrm{Au}, \mathrm{Pt}$; and various organic molecules ${ }^{25-27}$. Charge transfer from the intercalants ${ }^{7}$, or increased spin- 
53 orbit coupling owing to the presence of heavy atoms ${ }^{7,24,28}$, can enhance superconductivity ${ }^{10}$,

54 thermoelectricity ${ }^{25}$, or spin polarization ${ }^{7}$. The intercalation process typically involves post-growth, 55 diffusion-limited processes such as electrochemical or solid-state intercalation. A well-defined 56 intercalated phase with long-range crystalline order is difficult to obtain by such methods and usually 57 requires drastic treatment conditions ${ }^{29}$. Moreover, an intercalation phase diagram that correlates the 58 density and spatial distribution of intercalation atoms with mesoscopic properties of the intercalated 59 compound is currently lacking. Compared with foreign atom intercalation, intercalation of a TMD by its 60 native metal atoms has so far received scant attention ${ }^{11,29,30}$. Such self-intercalated TMD compounds may 61 exist as local energy minima in the part of the phase diagram where a metal-rich stoichiometry is 62 promoted by growth conditions using high chemical potential of metal atoms. However, to date, growth 63 windows of TMDs using high metal chemical potential remain relatively unexplored ${ }^{31,32}$.

64 In this work, we studied the growth of 2D TMDs under conditions of high metal chemical potential using 65 both molecular beam epitaxy (MBE) and chemical vapor deposition (CVD). We discovered that 66 independent of the employed growth method, a metal-rich chemical potential promotes the self67 intercalation of $\mathrm{M}$ into $\mathrm{MX}, \mathrm{MX}_{2}$, or $\mathrm{M}_{2} \mathrm{X}_{3}$ layered $2 \mathrm{D}$ compounds to produce a covalently bonded ic-2d $M_{x} X_{y}$ compound $\left(M=\right.$ metal, $X=$ chalcogen). Using $\operatorname{TaS}_{2}$ as an example, intercalated Ta atoms occupy the 69 octahedral vacancies in the vdW gap to form distinct topographical patterns, as verified by the atomic 70 resolution scanning transmission electron microscopy - annular dark field (STEM-ADF) imaging. By 71 varying the ratio of intercalated atoms to the octahedral vacancies in the vdW gap, we were able to grow $72 \mathrm{Ta}_{x} \mathrm{~S}_{y}$ or $\mathrm{Ta}_{x} \mathrm{Se}_{y}$ films with $\sigma \%$ of Ta-intercalation, where $\sigma \%$ refers to the ratio of occupied vacancy sites to 73 the initial total vacancy sites. Our results indicate that self-intercalation is generic to a broad class of vdW 74 crystals and it offers a powerful approach to transform layered 2D materials into ultrathin, covalently 75 bonded ic- $2 \mathrm{~d}$ crystals with ferromagnetic properties. 
77 We first describe the self-intercalation of a $\mathrm{TaS}_{2}$ bilayer by native atoms (i.e., Ta) during MBE deposition on a silicon wafer, this is used as an example to demonstrate the transformation of a 2D bilayer film into an ic-2d film via octahedral vacancy filling. Wafer-scale Ta-intercalated $\mathrm{TaS}_{2}$ bilayer films were grown on 2-inch $285 \mathrm{~nm} \mathrm{SiO}_{2} / \mathrm{Si}$ wafers in a dedicated MBE system ${ }^{14}$. Ultra-pure Ta and S molecular beams were evaporated from an e-beam evaporator and sulfur cracker cell equipped with a valve, respectively (Fig. 1a-b). We can routinely grow $2 \mathrm{H}$-phase $\mathrm{TaS}_{2}$ bilayer films using a high $\mathrm{S}$ chemical potential, i.e., Ta to $\mathrm{S}$ flux ratio 1:10 (Fig. 1a and Supplementary Fig. 1) for 3 hours, and a substrate temperature of $600{ }^{\circ} \mathrm{C}$. When the Ta:S flux ratio was increased to 1:6 (Fig. 1c), the film became non-stoichiometric with respect to $\mathrm{TaS}_{2}$ owing to the excess Ta atoms. A fingerprint of the Ta-rich environment is the presence of Ta adatoms (Fig. 1d) occupying the center of the honeycombs (highlighted in yellow) (Fig 1e) or atop of the

87 Ta sites (highlighted in white) (Fig. 1f) in monolayer $\mathrm{TaS}_{2}$ film, as observed by STEM when the growth was interrupted mid-way (Supplementary Fig. 2). By continually supplying Ta and $\mathrm{S}$ in the requisite ratio, the

89 Ta adatoms become embedded and occupy the octahedral vacancies between two S layers (Fig. 1g).

90 Therefore, the growth mechanism of ic- $2 \mathrm{~d}$ crystals follows a sequential $\mathrm{TaS}_{2}-\mathrm{Ta}_{\mathrm{a}}-\mathrm{TaS}_{2}$-Ta layer-by-layer

91 growth, such that multilayer or bulk phase ic- $2 \mathrm{~d}$ crystals can be readily accessed by simply increasing the 92 growth time. The thermodynamic stability of such intercalated phases is analyzed by the energy93 composition phase diagram generated through our DFT calculations (Fig. 1h).It can be seen that 94 stoichiometric $\mathrm{H}$-phase $\mathrm{TaS}_{2}$ is only formed under $\mathrm{S}$ rich conditions $\left(\mu_{\mathrm{s}}>-5.3 \mathrm{eV}\right)$, whereas under low $\mu_{\mathrm{s}}$, 95 various Ta-intercalated $\mathrm{Ta}_{x} \mathrm{~S}_{\mathrm{y}}$ configurations, ranging from $\mathrm{Ta}_{9} \mathrm{~S}_{16}$ (33.3\% Ta intercalation) to $\mathrm{Ta}_{9} \mathrm{~S}_{12}(100 \%$ 96 Ta intercalation), entered a thermodynamically stable state.

97 Notably, a 1:6 Ta:S ratio in the beam flux produced a $\sqrt{3} a \times \sqrt{3}$ a superlattice of Ta atoms (Fig. 2a) 98 sandwiched between two $\mathrm{TaS}_{2}$ monolayers. The coverage $\sigma$ was $33.3 \%$, and the overall stoichiometry of 
the crystal thus became $\mathrm{Ta}_{7} \mathrm{~S}_{12}$, as corroborated by both the real space STEM image (Fig. $2 \mathrm{~b}$ ) and the corresponding fast Fourier transform (FFT) pattern (Fig. 2c). Image simulation and sequential STEM images capturing the diffusion of intercalated atoms proved that the periodically arranged bright spots in the STEM image were induced by the Ta intercalation (see Fig. 2d, Supplementary Information section 1, and Supplementary Videos 1-2). We have also collected STEM cross-section image (Fig. 2e-f) to prove the existence of an intercalated Ta atomic layer in the vdW gap of ic-2d films grown by CVD.

The homogeneous $\operatorname{Ta}_{7} \mathrm{~S}_{12}$ phase was grown directly on a 2 -inch silicon wafer (Supplementary Fig. 3). The $\mathrm{Ta}_{7} \mathrm{~S}_{12}$ film was formed by coalescence of nano-domain crystals ( $\left.50 \mathrm{~nm}\right)$ separated by mirror twin boundaries or tilted grain boundaries (Supplementary Information section 2). Amorphous islands and gaps seen in the STEM images were due to the poor stability of $\mathrm{Ta}_{x} \mathrm{~S}_{y}$ and sample damage during the sample transfer. Energy dispersive x-ray spectroscopy (EDS) and electron energy loss (EEL) spectroscopy (Supplementary Fig. 4) verified that the chemical composition comprised solely of Ta and S with no foreign elements. X-ray photoelectron spectroscopy (XPS) (Supplementary Fig. 5) confirmed that the chemical stoichiometry agrees very well with $\mathrm{Ta}_{7} \mathrm{~S}_{12}$. The Raman spectra of the film exhibited two prominent $\mathrm{E}_{\mathrm{g}}^{3}$ and $A_{1 \mathrm{~g}}^{3}$ peaks at $300 \mathrm{~cm}^{-1}$ and $400 \mathrm{~cm}^{-1}$, respectively, matching those of $\mathrm{H}$-phase $\mathrm{TaS}_{2}$ films. The fingerprint of the intercalation came from a series of minor peaks in the $100 \mathrm{~cm}^{-1}$ to $170 \mathrm{~cm}^{-1}$ range (Supplementary Fig. 6), which were absent in pure $\mathrm{H}$-phase $\mathrm{TaS}_{2}{ }^{33}$ attributed to the covalent bonds between the intercalated Ta atoms and their octahedrally coordinated S atoms (Supplementary Fig. 7).

25\% Ta-intercalated $\mathrm{TaS}_{2}$ has a stoichiometry of $\mathrm{Ta}_{9} \mathrm{~S}_{16}$ and was produced at a slightly lowered Ta chemical potential, corresponding to a 1:8 Ta:S ratio. The intercalated Ta atoms occupy the octahedral vacancies in every $2 a \times \sqrt{3}$ a unit length, and this phase was distinguished by the square symmetry of the intercalated atomic lattice (Fig. 2g, 2k, and Supplementary Fig. 8). When the Ta:S flux ratio was further increased to $1: 5$, a $\mathrm{Ta}_{10} \mathrm{~S}_{16}$ phase $(\sigma=50 \%$ ) was successfully grown (Fig. $2 \mathrm{~h}$ ). The intercalation 
concentration was determined to be exactly 50\% via atom counting (Supplementary Fig. 9). Interestingly,

123 this phase is characterized by short-range interconnected atomic chains forming an overall glassy phase.

124 Clear diffusive rings were observed in the proximity of the first order FFT spots (Fig. 2l and Supplementary

125 Fig. 10), confirming the presence of this short-range ordered structure ${ }^{34}$. When we further enhanced the

126 Ta:S flux ratio, the glassy phase was retained, but the short Ta atomic chains became denser until it fully

127 evolved into a complete atomic plane when oreached $100 \%$ (Supplementary Fig. 11). When the growth

128 condition straddles between two high-symmetry phases, phase separations occur and atomically sharp

129 domain boundaries separating two high-symmetry phases can be clearly seen (Supplementary 130 Information section 3).

131 To verify that ic-2d films could be produced by methods other than MBE, we employed CVD to grow self132 intercalated $\mathrm{Ta}_{x} \mathrm{Se}_{y}$ crystals by using excess Ta precursors. The crystal domains of Ta-intercalated $\mathrm{Ta}_{x} \mathrm{Se}_{y}$ 133 films grown by CVD are in the micron-sized range, which are significantly larger than the nanosized domain 134 grown by MBE (Supplementary Fig. 12). A typical $\mathrm{Ta}_{8} \mathrm{Se}_{12}$ crystal $(\sigma=66.7 \%$ ) is depicted in Fig. $2 \mathrm{i}$. 135 Interestingly, it reveals a signature Kagome lattice belonging to the $\mathrm{P}_{6}$ wallpaper symmetry group. A well136 defined $\sqrt{3} a \times \sqrt{3}$ a periodic lattice can be unambiguously identified in the atomic-resolution STEM image 137 (Fig. $2 \mathrm{~m}$ and the simulated image in Supplementary Fig. 13). At even higher Ta chemical potential, $\mathrm{Ta}_{9} \mathrm{Se}_{12}$ 138 crystals $(\sigma=100 \%)$ were successfully synthesized, arising from the full occupation of the prismatic vacant 139 sites in $A A$-stacked Ta9 $\mathrm{Se}_{12}$ (Fig. 2j), as seen in the STEM top view (Fig. 2n) and side view images (Fig. 2e \& 140 Supplementary Fig. 14). By precisely controlling the metal:chalcogen ratio during the growth, a full 141 spectrum of Ta-intercalated $\mathrm{Ta}_{x} \mathrm{Se}_{y}$ or $\mathrm{Ta}_{x} \mathrm{~S}_{y}$ compounds with intercalation ratio ranging from $\sigma=25 \%$ to 142 over $100 \%$ can be grown, as further verified by EDS (Supplementary Fig. 15 \& Supplementary Table 1).

143 In the above example, the intercalated Ta atoms were octahedrally coordinated to the $\mathrm{S}_{6}$ cage as opposed 144 to the trigonal prismatic coordination adopted by pristine $\mathrm{TaS}_{2}$. Charge transfer from the intercalated Ta 
atoms to the $\mathrm{TaS}_{2}$ host layers creates new electron ordering and modified its $d$ band splitting. The

146 tunability in this system stems from the fact that the amount of charge transfer is dependent on the

147 intercalation concentration. To investigate if ferromagnetic order was present in the intercalated samples,

148 magneto-transport measurements were carried out on MBE-grown $\operatorname{Ta}_{7} \mathrm{~S}_{12}(\sigma=33.3 \%)$ with a 149 predominantly $2 \mathrm{H}_{\mathrm{a}}$ stacking registry (Fig. 3a and Supplementary Fig. 16) with bilayer thickness

150 (Supplementary Fig. 17). Fig. 3c shows the temperature-dependent resistivity, where a non-saturating 151 upturn is observed below $30 \mathrm{~K}$, due to the disorder-induced metal-insulator transition in the 152 polycrystalline sample ${ }^{35}$. Linear magneto-resistance (MR) up to $9 \mathrm{~T}$ at low temperature is observed in $153 \mathrm{Ta}_{7} \mathrm{~S}_{12}$ (Fig. 3d) due to the density and mobility fluctuations ${ }^{36}$. The anomalous Hall effect (AHE) arises from 154 the interplay of spin-orbit interactions and ferromagnetic order, and is a potentially useful probe of spin 155 polarization. We observed $\mathrm{AHE}$ in $\mathrm{Ta}_{7} \mathrm{~S}_{12}$ on top of the linear ordinary Hall effect (OHE). Fig. 3e shows the 156 nonlinear Hall effect at the proximity of zero magnetic field and linear OHE at high field. While both 157 multiband conduction and AHE contribute to nonlinear Hall effect, the observed linear OHE suggests 158 single carrier conduction (hole) in $\mathrm{Ta}_{7} \mathrm{~S}_{12}$ and thus excludes multiband transport as the origin of the 159 nonlinear Hall effect ${ }^{37,38}$. The nonlinear Hall effect is thus ascribed to AHE, which stems from 160 ferromagnetism in conductors ${ }^{39}$. After subtracting the linear $\mathrm{OHE}$, anomalous hall resistance up to $0.75 \Omega$ 161 is observed at $1.5 \mathrm{~K}$; it decreases with increasing temperature and vanishes at $10 \mathrm{~K}$ in line with Monte 162 Carlo simulation based on Ising model (Supplementary Fig. 18).

163 The effects of self-intercalation on the electrical properties of TMDCs are further tested on the Kagome 164 lattice $\mathrm{Ta}_{8} \mathrm{Se}_{12}(\sigma=66.7 \%)$. It was observed that the intercalation of $\mathrm{Ta}$ atoms and formation of Kagome 165 lattices stabilize the charge density wave (CDW) states. Temperature dependent Hall signal reveals AHE 166 below $15 \mathrm{~K}$ and confirms the ferromagnetic order in $\mathrm{Ta}_{8} \mathrm{Se}_{12}$ (Supplementary Fig. 19-20). 
167 We performed DFT calculations to gain an understanding of the origin of the magnetization in self168 intercalated $\mathrm{Ta}_{7} \mathrm{~S}_{12}$. Perfect bilayer $2 \mathrm{H}_{\mathrm{a}}$-stacked $\mathrm{TaS}_{2}$ (Supplementary Fig. 21) possesses a non-magnetic 169 ground state. Ferromagnetism can be induced by the double exchange mechanism ${ }^{40}$ triggered by the 170 charge transfer from intercalated Ta to pristine $\mathrm{TaS}_{2}$ (Fig. 3f). When the intercalated Ta adopts a $\sqrt{3} \mathrm{a} \times \sqrt{3} \mathrm{a}$ 171 superstructure, six S atoms bond with one intercalated Ta atom to form an octahedral unit in the vdW 172 gap. In contrast, each S atom is shared by three Ta atoms in the pristine $\mathrm{TaS}_{2}$ layer. This difference in local 173 bonding arrangement induces charge transfer from the octahedral-coordinated intercalated Ta atom to 174 the prismatic-coordinated Ta atom in the $\mathrm{TaS}_{2}$ layer (Fig. 3f). In the pristine H-phase TaS 2 , the Ta $d$ and S $175 p$ orbitals are well separated in terms of energy, with the states at the Fermi level having mainly Ta $d_{z^{2}}$ 176 and Ta $d_{x^{2}}$ characteristics (Supplementary Fig. 21). In $\mathrm{Ta}_{7} \mathrm{~S}_{12}(\sigma=33.3 \%)$, the intercalated Ta atoms 177 introduce additional spin-split bands across the Fermi level, and a magnetic ground state develops(Fig. $1783 g-h)$. The magnetic moments are localized on the $d$ orbitals of the intercalated Ta atom, as evidenced by 179 the calculated orbital-resolved spin up and spin down band structures in Fig. 3g and Fig. 3h, respectively. 180 The states at the Fermi level comprise the prismatic-centred Ta $d_{z^{2}}$ orbitals hybridized with the spin-up 181 band of the $d_{x^{2}-y^{2}}$ orbital of the intercalated Ta. However, only the intercalated Ta atoms exhibit a net 182 spin density, as illustrated in Fig. 3i, where the top view spin density isosurface matches the shape of the $183 d_{x^{2}-y^{2}}$ orbital. In addition, the non-magnetic 3a $\times 3$ 3a CDW state of $\mathrm{Ta}_{7} \mathrm{~S}_{12}$ can be ruled out owing to its 184 relative instability compared to the ferromagnetic state ${ }^{41}$.

185 The existence of a magnetic moment correlates with a strong charge transfer between the intercalated $186 \mathrm{Ta}$ and the $\mathrm{TaS}_{2}$ layers. Strong charge transfer occurs in the dilute Ta-intercalated compound, whereas the 187 charge transfer becomes relatively weak in a heavily intercalated (Fig. 3j) compound in accordance with 188 the calculated charge difference and the variation of Bader charge in the Ta atoms (Supplementary Table 2, \& Supplementary Fig. 22). 
To investigate whether the self-intercalation phenomenon occurred for other TMDs, we performed a high-throughput DFT study of 48 different intercalated TMD bilayers using a semi-automated workflow for maximal consistency and veracity ${ }^{42}$. Specifically, we considered TMDs of the transition metals Mo, W, $\mathrm{Nb}, \mathrm{Ta}, \mathrm{Ti}, \mathrm{Zr}, \mathrm{Hf}, \mathrm{V}, \mathrm{Cr}, \mathrm{Mn}, \mathrm{Fe}, \mathrm{Co}, \mathrm{Ni}, \mathrm{Pd}, \mathrm{Pt}$, and $\mathrm{Sn}$, and the chalcogens $\mathrm{S}$, Se, and Te (highlighted in Fig. 4a in blue and red, respectively) when $\sigma$ equals $33.3 \%$ or $66.7 \%$. Out of this set of TMDs, we observed that 14 bilayer configurations, $\mathrm{Ti}_{8} \mathrm{~S}_{12}, \mathrm{Ti}_{8} \mathrm{Se}_{12}, \mathrm{Ti}_{8} \mathrm{Te}_{12}, \mathrm{Co}_{7} \mathrm{~S}_{12}, \mathrm{Co}_{7} \mathrm{Se}_{12}, \mathrm{Co}_{7} \mathrm{Te}_{12}, \mathrm{Nb}_{7} \mathrm{~S}_{12}, \mathrm{Nb}_{7} \mathrm{Se}_{12}, \mathrm{Nb}_{7} \mathrm{Te}_{12}, \mathrm{Mo}_{7} \mathrm{~S}_{12}$, $\mathrm{Mo}_{7} \mathrm{Se}_{12}, \mathrm{Ta}_{7} \mathrm{~S}_{12}, \mathrm{Ta}_{7} \mathrm{Se}_{12}$, and $\mathrm{Ta}_{7} \mathrm{Te}_{12}$, (highlighted in Fig. 4a and Supplementary Table 3 for magnetic moment) develop ferromagnetic order upon self-intercalation, whereas their parental $\mathrm{MX}_{2}$ are nonferromagnetic. Notably, group V and VI TMDs, exhibit strong ferromagnetism after self-intercalation (Fig. 4b). Intrinsic ferromagnetic $\mathrm{MX}_{2}\left(\mathrm{VX}_{2}, \mathrm{CrX}_{2}, \mathrm{MnX}_{2}\right.$, and $\left.\mathrm{FeX}_{2}\right)$ retain ferromagnetism upon selfintercalation (highlighted in Fig. 4a in orange). Among the 14 types of self-intercalated 2D ferromagnets, the formation energies of 12 of them (the 2 exceptions being $\mathrm{MoS}_{2}$ and $\mathrm{MoSe}_{2}$ ) were lower than or similar to those of the non-intercalated materials (Supplementary Fig. 23-24), indicating that self-intercalation is energetically feasible.

To validate the theoretical predictions, we attempted to grow a wide variety of $i c-2 \mathrm{~d}$, as highlighted by the triangle in the top left corner in Fig. 4a. Blue triangles indicate that the self-intercalation can be experimentally realized ${ }^{11,12}$, whereas gray triangles indicate that intercalation was not be successful under our experimental conditions. We had succeeded to grow several ic- $2 \mathrm{~d}$ crystals, namely $\mathrm{V}_{11} \mathrm{~S}_{16}$ (Fig. $4 \mathrm{c}$ and Supplementary Fig. 25), $\ln _{11} \mathrm{Se}_{16}$ (Fig. $4 \mathrm{~d}$ and Supplementary Fig. 26), and $\mathrm{Fe}_{\mathrm{x}} \mathrm{Te}_{\mathrm{y}}$ (Fig. 4e and Supplementary Fig. 27) by CVD or MBE. Their clear topological features and corresponding FFT patterns are depicted in Fig. $4 \mathrm{f}-\mathrm{h}$. The intercalated $\mathrm{V}_{11} \mathrm{~S}_{16}$ revealed a $2 \mathrm{a} \times 2 \mathrm{2}$ superstructure, and the intercalation ratio was estimated at $75 \%$ (Fig. 4 f). $\ln _{11} \mathrm{Se}_{16}$ also showed a $2 \mathrm{a} \times 2 \mathrm{a}$ superstructure, but in this case, the intercalated In atoms reveal a signature honeycomb structure (Fig. 4g). The crystal structure of selfintercalated $\mathrm{Fe}_{x} \mathrm{Te}_{y}$ was rather complicated with additional Fe intercalated into the atomic network of the 
214 pristine FeTe matrix as interstitials because telluride based TMDs offer the largest spacing between the

215 host atoms ${ }^{43}$. Upon intercalation, the novel $\mathrm{Fe}_{\mathrm{x}} \mathrm{Te} \mathrm{y}$ phase reveals new symmetries as confirmed by the 216 emergence of superspots in the FFT pattern (Fig. 4h). Similar complex intercalation network has also been 217 observed in $\mathrm{V}_{\mathrm{x}} \mathrm{Te}_{\mathrm{y}}$ (Supplementary Fig. 28).

\section{Conclusion}

219 We have discovered a robust way to perform composition engineering of a broad class of TMD by self220 intercalation with native metal atoms during growth. The main principle is to apply a high chemical 221 potential of metal atoms to provide the driving force for intercalation during growth, thus it should work

222 for most growth methods. The metal intercalants occupy octahedral vacant sites in the vdW gap, and 223 depending on the coverage patterns, distinct stoichiometric phases are produced. High throughput DFT 224 simulations, supported by growth experiments, show that the self-intercalation method is applicable to a 225 large class of 2D layered materials, thus allowing a whole new library of materials with potentially new 226 properties to be created from existing layered materials. Owing to the versatility in composition control, 227 it is possible to tune in one class of materials, properties that can vary from ferromagnetic to non228 ferromagnetic, and spin-frustrated Kagome lattices. In particular, ferromagnetic order can be introduced 229 into non-magnetic layered materials via the self-intercalation approach, giving rise to a unique topological 230 phase such as ferromagnetic Kagome lattices. The implication of this work is that bilayer TMD (or thicker)

231 can be transformed into ultrathin covalent bonded 3D material, with a stoichiometry that is tuneable by 232 the concentration of the intercalants over a broad range.

\section{References}

234 1. Chhowalla, M. et al. The chemistry of two-dimensional layered transition metal dichalcogenide 235 nanosheets. Nature Chemistry 5, 263-275 (2013).

236 2. Zhou, J. et al. A library of atomically thin metal chalcogenides. Nature 556, 355-359 (2018). 
3. Jin, C. et al. Ultrafast dynamics in van der Waals heterostructures. Nature Nanotechnology 13, 994-1003 (2018).

4. Wang, C. et al. Monolayer atomic crystal molecular superlattices. Nature 555, 231-236 (2018).

5. Novoselov, K. S. et al. Two-dimensional atomic crystals. Proc. Natl. Acad. Sci. U. S. A. 102, 1045110453 (2005).

6. Wan, J. et al. Tuning two-dimensional nanomaterials by intercalation: Materials, properties and applications. Chemical Society Reviews 45, 6742-6765 (2016).

7. Friend, R. H. \& Yoffe, A. D. Electronic properties of intercalation complexes of the transition metal dichalcogenides. Adv. Phys. 36, 1-94 (1987).

8. Wang, X., Shen, X., Wang, Z., Yu, R. \& Chen, L. Atomic-scale clarification of structural transition of MoS2 upon sodium intercalation. ACS Nano 8, 11394-11400 (2014).

9. Tan, S. J. R. et al. Chemical Stabilization of 1T' Phase Transition Metal Dichalcogenides with Giant Optical Kerr Nonlinearity. J. Am. Chem. Soc. 139, 2504-2511 (2017).

10. Kanetani, K. et al. Ca intercalated bilayer graphene as a thinnest limit of superconducting C6Ca. Proc. Natl. Acad. Sci. U. S. A. 109, 19610-19613 (2012).

11. Yang, J. et al. Ultrahigh-current-density niobium disulfide catalysts for hydrogen evolution. Nat. Mater. (2019). doi:10.1038/s41563-019-0463-8

12. Cui, F. et al. Controlled Growth and Thickness-Dependent Conduction-Type Transition of 2D Ferrimagnetic Cr2S3 Semiconductors. Adv. Mater. 32, 1905896 (2020).

13. Mortazavi, M., Wang, C., Deng, J., Shenoy, V. B. \& Medhekar, N. V. Ab initio characterization of layered MoS2 as anode for sodium-ion batteries. J. Power Sources 268, 279-286 (2014).

14. Fu, D. et al. Molecular Beam Epitaxy of Highly Crystalline Monolayer Molybdenum Disulfide on Hexagonal Boron Nitride. J. Am. Chem. Soc. 139, 9392-9400 (2017).

15. Chen, J. et al. Homoepitaxial Growth of Large-Scale Highly Organized Transition Metal Dichalcogenide Patterns. Adv. Mater. 30, 1704674 (2018).

16. Liao, M. et al. Twist angle-dependent conductivities across MoS2/graphene heterojunctions. Nat. Commun. 9, 4068 (2018).

17. Koski, K. J. et al. Chemical intercalation of zerovalent metals into 2D layered Bi2Se 3 nanoribbons. J. Am. Chem. Soc. 134, 13773-13779 (2012).

18. Guilmeau, E., Barbier, T., Maignan, A. \& Chateigner, D. Thermoelectric anisotropy and texture of intercalated TiS2. Appl. Phys. Lett. 111, 133903 (2017). 
19. Wang, M. et al. Chemical intercalation of heavy metal, semimetal, and semiconductor atoms into 2D layered chalcogenides. 2D Mater. 5, 045005 (2018).

20. Dungey, K. E., Curtis, M. D. \& Penner-Hahn, J. E. Structural Characterization and Thermal Stability of MoS2 Intercalation Compounds. Chem. Mater. 10, 2152-2161 (1998).

21. Gong, Y. et al. Spatially controlled doping of two-dimensional SnS2 through intercalation for electronics. Nat. Nanotechnol. 13, 294-299 (2018).

22. Chen, Z. et al. Interface confined hydrogen evolution reaction in zero valent metal nanoparticlesintercalated molybdenum disulfide. Nat. Commun. 8, 14548 (2017).

23. Liu, C. et al. Dynamic Ag+-intercalation with AgSnSe2 nano-precipitates in Cl-doped polycrystalline SnSe2 toward ultra-high thermoelectric performance. J. Mater. Chem. A 7, 97619772 (2019).

24. Bouwmeester, H. J. M., Van Der Lee, A., Van Smaalen, S. \& Wiegers, G. A. Order-disorder transition in silver-intercalated niobium disulfide compounds. II. Magnetic and electrical properties. Phys. Rev. B 43, 9431-9435 (1991).

25. Wan, C. et al. Flexible n-type thermoelectric materials by organic intercalation of layered transition metal dichalcogenide TiS2. Nat. Mater. 14, 622-627 (2015).

26. Jeong, S. et al. Tandem intercalation strategy for single-layer nanosheets as an effective alternative to conventional exfoliation processes. Nat. Commun. 6, 5763 (2015).

27. O'Brien, E. S. et al. Single-crystal-to-single-crystal intercalation of a low-bandgap superatomic crystal. Nat. Chem. 9, 1170-1174 (2017).

28. Kumar, P., Skomski, R. \& Pushpa, R. Magnetically Ordered Transition-Metal-Intercalated WSe2. ACS Omega 2, 7985-7990 (2017).

29. Kim, S. et al. Interstitial Mo-Assisted Photovoltaic Effect in Multilayer MoSe2 Phototransistors. Adv. Mater. 30, 1705542 (2018).

30. Zhang, M. et al. Electron Density Optimization and the Anisotropic Thermoelectric Properties of Ti Self-Intercalated Ti1+xS2 Compounds. ACS Appl. Mater. Interfaces 10, 32344-32354 (2018).

31. Wang, S. et al. Shape evolution of monolayer MoS2 crystals grown by chemical vapor deposition. Chem. Mater. 26, 6371-6379 (2014).

32. Zhao, X. et al. Mo-Terminated Edge Reconstructions in Nanoporous Molybdenum Disulfide Film. Nano Lett. 18, 482-490 (2018).

33. Mounet, N. et al. Two-dimensional materials from high-throughput computational exfoliation of experimentally known compounds. Nat. Nanotechnol. 13, 246-252 (2018). 
34. Azizi, A. et al. Spontaneous Formation of Atomically Thin Stripes in Transition Metal Dichalcogenide Monolayers. Nano Lett. 16, 6982-6987 (2016).

35. Motome, Y., Furukawa, N. \& Nagaosa, N. Competing Orders and Disorder-Induced Insulator to Metal Transition in Manganites. Phys. Rev. Lett. 91, 167204 (2003).

36. Parish, M. M. \& Littlewood, P. B. Non-saturating magnetoresistance in heavily disordered semiconductors. Nature 426, 162-165 (2003).

37. Jiang, Z. et al. Structural and proximity-induced ferromagnetic properties of topological insulatormagnetic insulator heterostructures. AIP Adv. 6, 55809 (2016).

38. Jiang, Z. et al. Independent Tuning of Electronic Properties and Induced Ferromagnetism in Topological Insulators with Heterostructure Approach. Nano Lett. 15, 5835-5840 (2015).

39. Nagaosa, N., Sinova, J., Onoda, S., MacDonald, A. H. \& Ong, N. P. Anomalous Hall effect. Rev. Mod. Phys. 82, 1539-1592 (2010).

40. Zener, C. Interaction between the d shells in the transition metals. Phys. Rev. 81, 440-444 (1951).

41. Coelho, P. M. et al. Charge Density Wave State Suppresses Ferromagnetic Ordering in VSe2 Monolayers. J. Phys. Chem. C 123, 14089-14096 (2019).

42. Haastrup, S. et al. The Computational 2D Materials Database: High-throughput modeling and discovery of atomically thin crystals. 2D Materials 5, 042002 (2018).

43. Karthikeyan, J., Komsa, H.-P., Batzill, M. \& Krasheninnikov, A. V. Which Transition Metal Atoms Can Be Embedded into Two-Dimensional Molybdenum Dichalcogenides and Add Magnetism? Nano Lett. 19, 4581-4587 (2019).

44. Wang, H. et al. High-quality monolayer superconductor $\mathrm{NbSe} 2$ grown by chemical vapour deposition. Nat. Commun. 8, 394 (2017).

45. Enkovaara, J. et al. Electronic structure calculations with GPAW: A real-space implementation of the projector augmented-wave method. Journal of Physics Condensed Matter 22, 253202 (2010).

46. Monkhorst, H. J. \& Pack, J. D. Special points for Brillouin-zone integrations. Phys. Rev. B 13, 51885192 (1976).

47. Perdew, J. P., Burke, K. \& Ernzerhof, M. Generalized gradient approximation made simple. Phys. Rev. Lett. 77, 3865-3868 (1996).

48. Wellendorff, J. et al. Density functionals for surface science: Exchange-correlation model development with Bayesian error estimation. Phys. Rev. B 85, 235149 (2012). 
K. P. L thanks A*STAR Project "Scalable Growth of Ultrathin Ferroelectric Materials for Memory Technologies, grant number A1983c0035", and support from Centre for Advanced 2D Materials, NUS. W.Z. acknowledges support from the National Key R\&D Program of China (2018YFA0305800) and the Natural Science Foundation of China (51622211). S. J. P. is grateful to the National University of Singapore for funding and MOE for a Tier 2 grant "Atomic scale understanding and optimization of defects in 2D materials" (MOE2017-T2-2-139). Z. L. thanks MOE for a Tier 2 grant (2017-T2-2-136), Tier 3 grant (2018T3-1-002), and A*Star QTE programme. X. L. thanks support from National Natural Science Foundation of China (Grant No: 11804286) and the Fundamental Research Funds for the Central Universities (Grant No 19|gpy263), X. L. acknowledges the support from the Rosamond Center for Computation and Visualization in School of Physics at Sun Yat-sen University. K. S. T. acknowledge funding from the European Research Council (ERC) under the European Union's Horizon 2020 research and innovation programme (Grant No. 773122, LIMA). The Center for Nanostructured Graphene is sponsored by the Danish National Research Foundation, Project DNRF103. The authors thank J. P. Shi., F. F. Cui., and Y. F. Zhang for providing highquality CVD samples.

\section{Author contributions}

X. Z., S. J. P., and K. P. L. conceived the idea. S. J. P., and K. P. L. supervised the execution of the whole work. X. Z. performed the electron microscopy experiments and data analysis. X. L., A. C. R. and C. C. W. did the DFT calculation and data analysis. A. C. R-J. and K. S. T. performed the high-throughput DFT calculations. W. F., Y. D., and L. K. grew the samples. D. W. measured the magnetism. P. S. did device fabrication and measurement. J. D., and S. N. developed the python scripts for data analysis. All the authors discussed the results. All authors participated in writing the manuscripts. X. Z., and P. S. contributed equally to this work.

\section{Competing interests}

356 The authors declare no conflict of interest.

\section{Additional information}

Supplementary information is available for this paper at

Reprints and permissions information is available at www. nature.com/reprints.

Correspondence and requests for materials should be addressed to X. L., S. J. P. and K. P. L.

Publisher's note: Springer Nature remains neutral with regard to jurisdictional claims in published maps and institutional affiliations. 

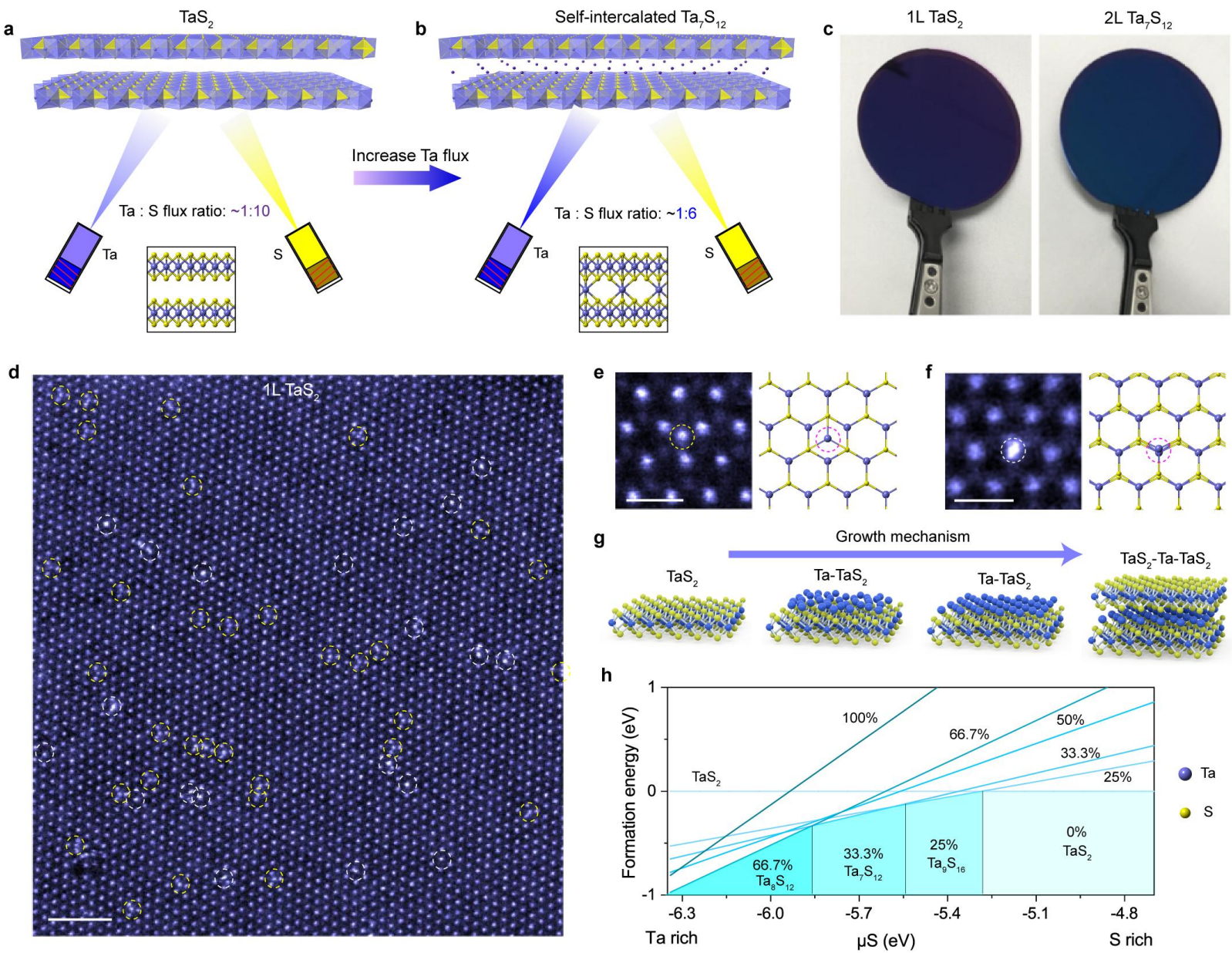

Figure 1. Self-intercalation in $\mathrm{TaS}_{2}$ crystals. Schematic illustration showing the MBE growth of (a) pristine

$367 \mathrm{TaS}_{2}$ and (b) self-intercalated $\mathrm{Ta}_{7} \mathrm{~S}_{12}$ under a low and high Ta-flux environment, respectively. The lower Ta

368 flux produces stoichiometric $\mathrm{TaS}_{2}$, whereas higher Ta flux leads to a self-intercalated phase. (c)

369 Photographs of MBE-grown $1 \mathrm{~L}-\mathrm{TaS}_{2}$ and $2 \mathrm{~L}-\mathrm{Ta}_{7} \mathrm{~S}_{12}$ on 2-inch $\mathrm{SiO}_{2} / \mathrm{Si}$ wafer. (d) Atomic-resolution STEM-

370 ADF image of monolayer $\mathrm{TaS}_{2}$ under Ta rich conditions with abundant Ta interstitials locating at the (e)

371 center of honeycomb or (f) on top of the Ta site. Corresponding atomic models were depicted in the right

372 panels. (g) Schematic illustration depicting the layer-by-layer growth of ic-2d crystals. (h) Calculated

373 formation energies of various self-intercalated $\mathrm{Ta}_{x} \mathrm{~S}_{y}$ phases where the intercalation concentrations are 

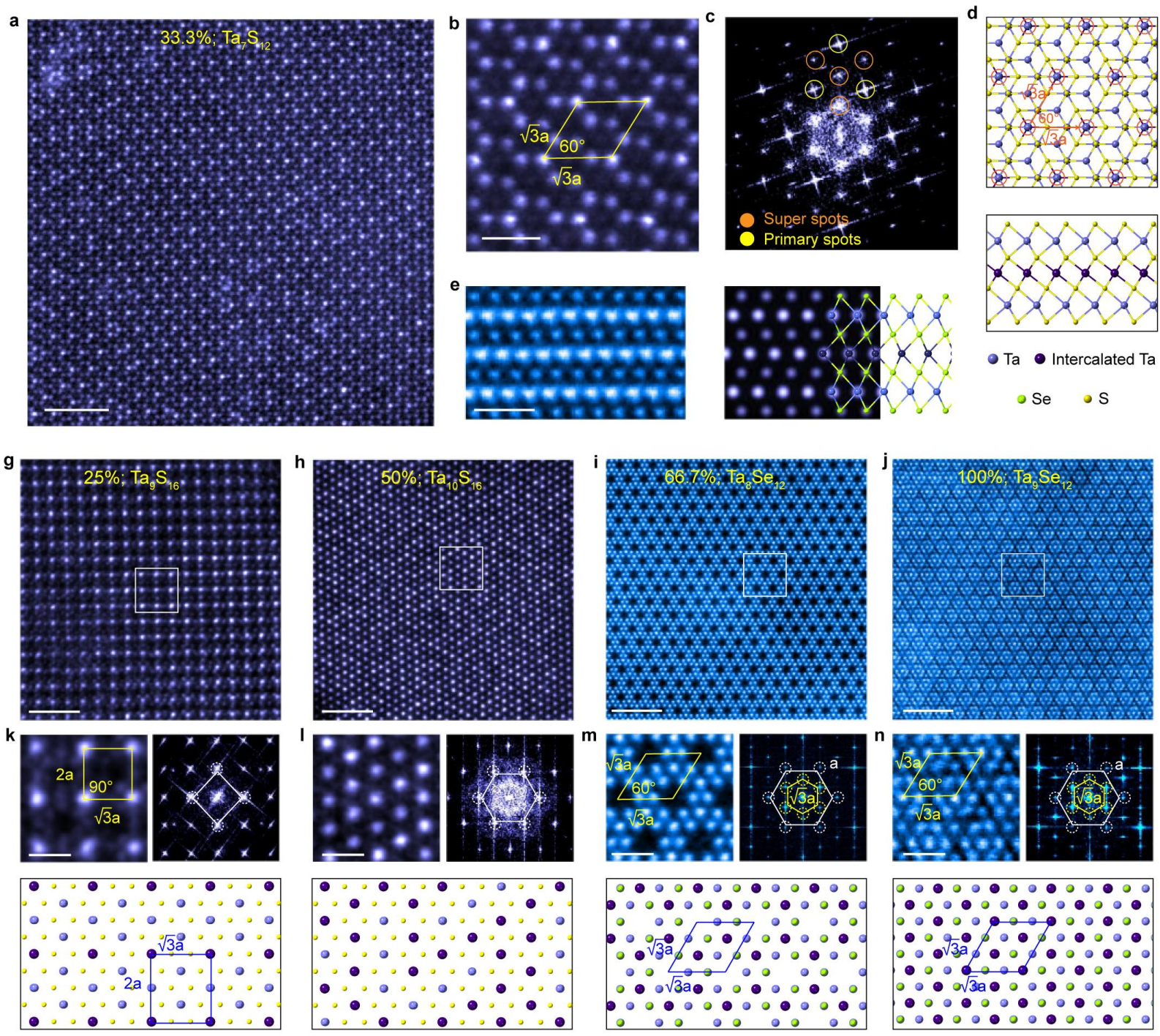

Figure 2. Compositional engineering of self-intercalated $\mathrm{Ta}_{x} \mathrm{~S}_{y}\left(\mathrm{Ta}_{x} \mathrm{Se}_{y}\right)$ with different intercalated $\mathrm{Ta}$ 

STEM images of (g) $25 \%$ self-intercalated $\mathrm{Ta}_{9} \mathrm{~S}_{16}$, (h) $50 \%$ self-intercalated $\mathrm{Ta}_{10} \mathrm{~S}_{16}$, (i) $66.7 \%$ selfintercalated $\mathrm{Ta}_{8} \mathrm{Se}_{12}$, and (j) $100 \%$ self-intercalated $\mathrm{Ta}_{9} \mathrm{Se}_{12}$ ic-2d crystals. (k-n) Enlarged white box regions

385 from (g-j), respectively, and their corresponding FFT patterns and atomic models are depicted in the right, 386 and lower panels, respectively. Scale bars: $2 \mathrm{~nm}$ in (a, g-j); $0.5 \mathrm{~nm}$ in (b, e, k-n).
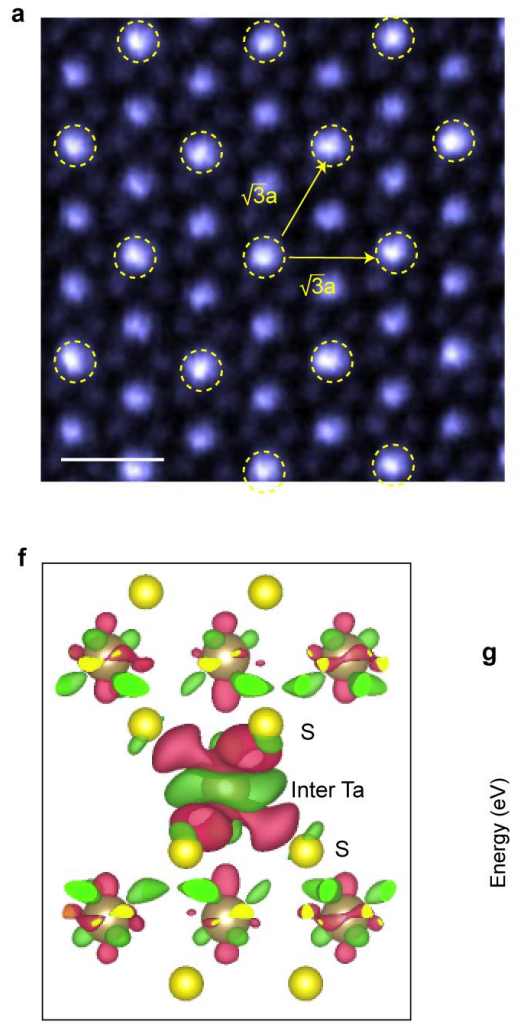
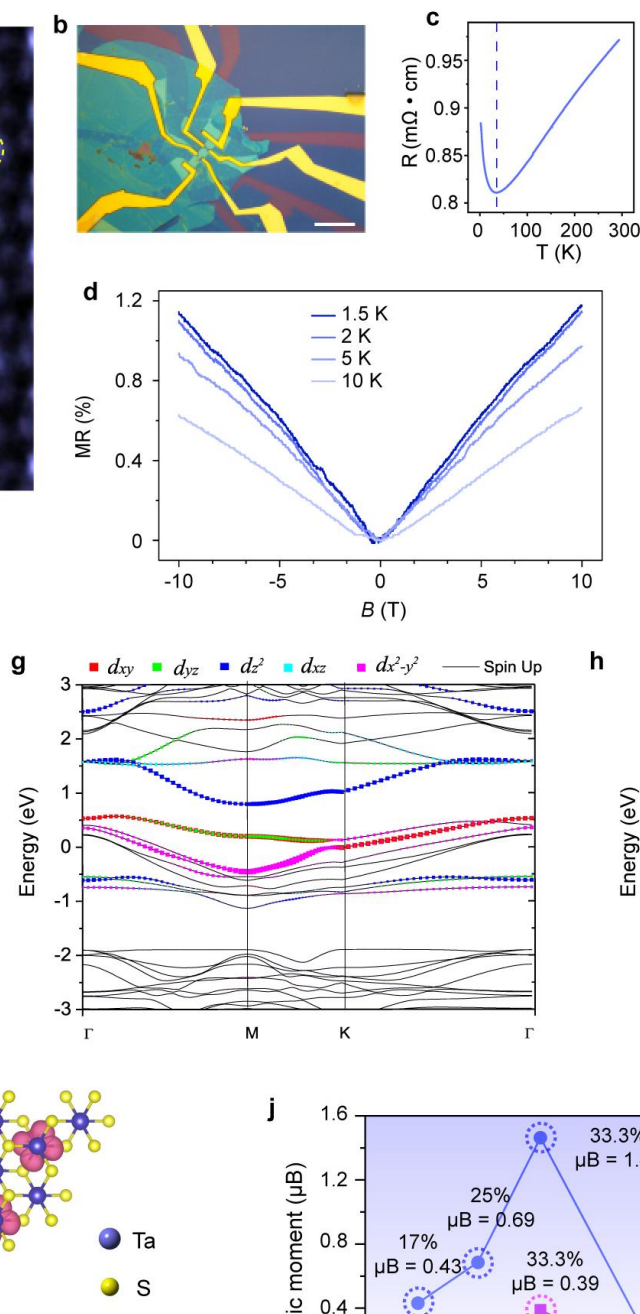
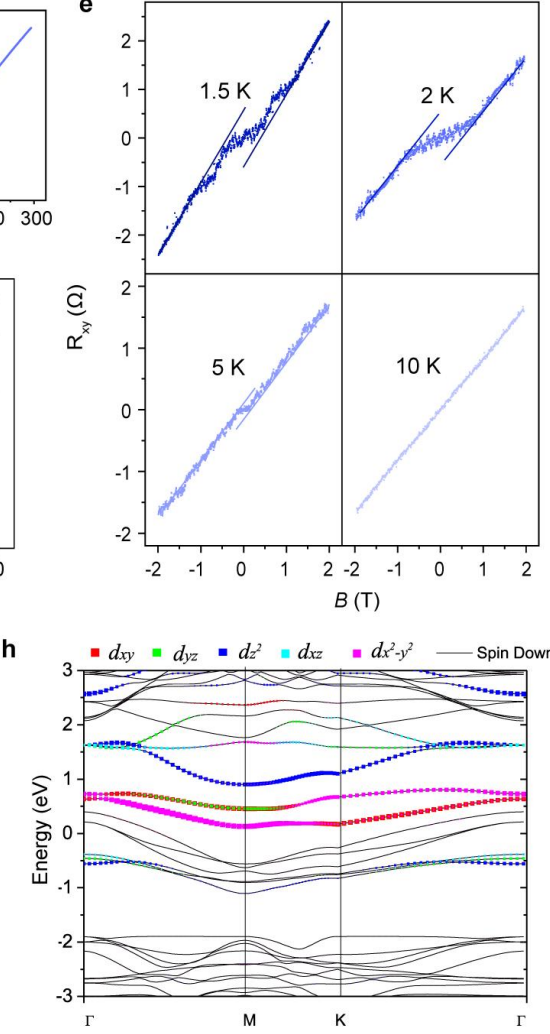

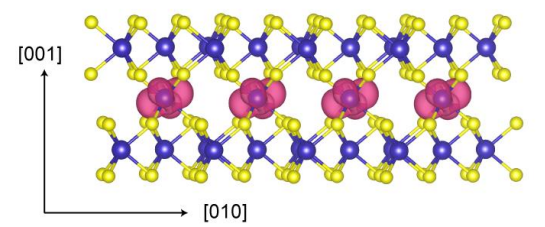

i

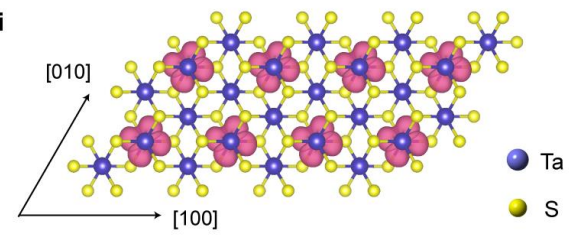

387

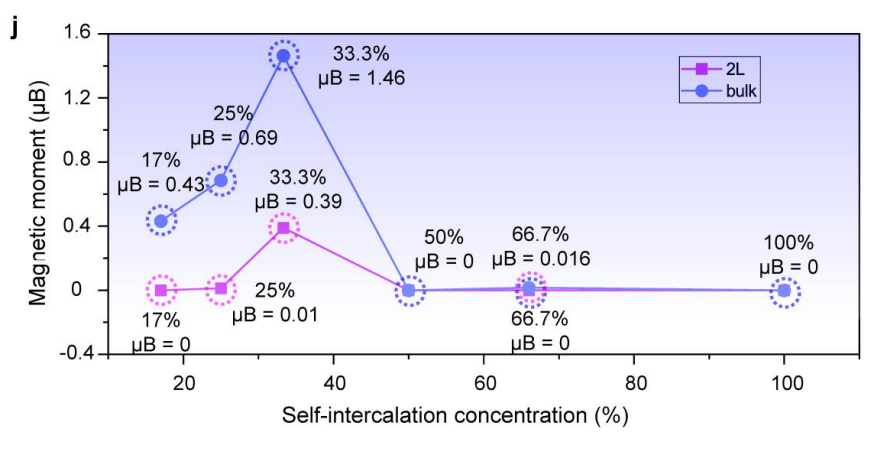


388 Figure 3. Ferromagnetism in self-intercalated $\mathrm{Ta}_{7} \mathbf{S}_{12}$ ic-2d crystals. (a) Atomic-resolution STEM-ADF 389 image of a typical self-intercalated $\mathrm{Ta}_{7} \mathrm{~S}_{12}$ film. (b) Optical microscopy image of $\mathrm{Ta}_{7} \mathrm{~S}_{12}$ Hall bar device 390 encapsulated with $h$-BN. (c) Resistivity as a function of temperature. Temperature dependent (d) 391 longitudinal resistance $\left(R_{x x}\right)$ and (e) Hall resistance $\left(R_{x y}\right)$ under the out-of-plane magnetic field. (f) Contour 392 plot of charge density difference in Ta-intercalated $\mathrm{Ta}_{7} \mathrm{~S}_{12}$. Orbital-resolved (g) spin up and (h) spin down 393 band structures of $\mathrm{Ta}_{7} \mathrm{~S}_{12}$. (i) Top view and the side view spin density isosurface of Ta-intercalated Ta7 $\mathrm{S}_{12}$.

394 (j) Calculated magnetic moments as a function of Ta intercalation concentration in nonstoichiometric $\mathrm{Ta}_{x} \mathrm{~S}_{\mathrm{y}}$. 395 The STEM-ADF image shown in Fig. 3a was collected using a half-angle range from 30 to $110 \mathrm{mrad}$ to 396 enhance the contrast of S. Scale bar: $0.5 \mathrm{~nm}$ in (a); $20 \mu \mathrm{m}$ in (b). 


\begin{tabular}{|c|c|c|c|c|c|c|c|c|c|c|c|c|c|c|}
\hline$\| \mathrm{A}$ & & & & & & & & & & & & & VIA & VIIA \\
\hline $\mathrm{Mg}$ & & & & & & & & & & & & & $S$ & $\mathrm{Cl}$ \\
\hline 12 & IIIB & IVB & VB & VIB & VIIB & & $-\mathrm{VIII}$ & & $\mathrm{AB}$ & $\| \mathrm{B}$ & $\| \mathrm{IIA}$ & IVA & 16 & 17 \\
\hline $\begin{array}{l}\mathrm{Ca} \\
20\end{array}$ & $\begin{array}{l}\text { Sc } \\
22\end{array}$ & $\begin{array}{l}\mathrm{Ti} \\
22 \mathrm{Se} \\
\mathrm{Ti}\end{array}$ & $\begin{array}{l}\mathrm{V} \\
23\end{array}$ & $\begin{array}{l}\mathrm{Cr} \\
24\end{array}$ & $\begin{array}{c}\mathrm{Mn} \\
25\end{array}$ & $\begin{array}{c}\mathrm{Fe} \\
26\end{array}$ & $\begin{array}{c}\mathrm{Co}_{\mathrm{S}}^{33.3 \%} \\
27 \mathrm{Se} \\
\mathrm{Te}\end{array}$ & $\begin{array}{l}\mathrm{Ni} \\
28\end{array}$ & $\begin{array}{l}\mathrm{Cu} \\
29\end{array}$ & $\begin{array}{l}\mathrm{Zn} \\
30\end{array}$ & $\begin{array}{c}\mathrm{Ga} \\
31\end{array}$ & $\begin{array}{c}\mathrm{Ge} \\
32\end{array}$ & $\begin{array}{c}\mathrm{Se} \\
34\end{array}$ & $\begin{array}{l}\mathrm{Br} \\
35\end{array}$ \\
\hline $\begin{array}{l}\mathrm{Sr} \\
38\end{array}$ & $\begin{array}{c}Y \\
39\end{array}$ & $\begin{array}{l}\mathrm{Zr} \\
40\end{array}$ & $\begin{array}{c}333 \% \\
\mathrm{Nb} \\
41 \mathrm{se} \\
\mathrm{Te}\end{array}$ & $\begin{array}{l}\mathrm{Mo}^{33.3 \%} \\
42 \mathrm{~S}\end{array}$ & $\begin{array}{l}\text { Tc } \\
43\end{array}$ & $\begin{array}{c}\mathrm{Ru} \\
44\end{array}$ & $\begin{array}{l}\mathrm{Rh} \\
45\end{array}$ & $\begin{array}{c}P d \\
46\end{array}$ & $\begin{array}{c}\mathrm{Ag} \\
47\end{array}$ & $\begin{array}{l}\mathrm{Cd} \\
48\end{array}$ & In & $\begin{array}{l}\text { Sn } \\
50\end{array}$ & $\begin{array}{l}\mathrm{Te} \\
52\end{array}$ & $\begin{array}{c}\text { I } \\
53\end{array}$ \\
\hline & $\begin{array}{l}\text { La } \\
57\end{array}$ & $\begin{array}{l}\mathrm{Hf} \\
72\end{array}$ & $\begin{array}{l}333.6 \\
\mathrm{Ta}^{33} \\
73 \mathrm{Se}\end{array}$ & $\begin{array}{l}\text { W } \\
74\end{array}$ & $\begin{array}{c}\mathrm{Re} \\
75\end{array}$ & $\begin{array}{l}\text { Os } \\
76\end{array}$ & $\begin{array}{l}\text { Ir } \\
77\end{array}$ & $\begin{array}{l}\mathrm{Pt} \\
78\end{array}$ & $\begin{array}{c}\mathrm{Au} \\
79\end{array}$ & $\begin{array}{c}\mathrm{Hg} \\
80\end{array}$ & $\begin{array}{l}\text { TI } \\
81\end{array}$ & $\begin{array}{c}\mathrm{Pb} \\
82\end{array}$ & & \\
\hline
\end{tabular}

b
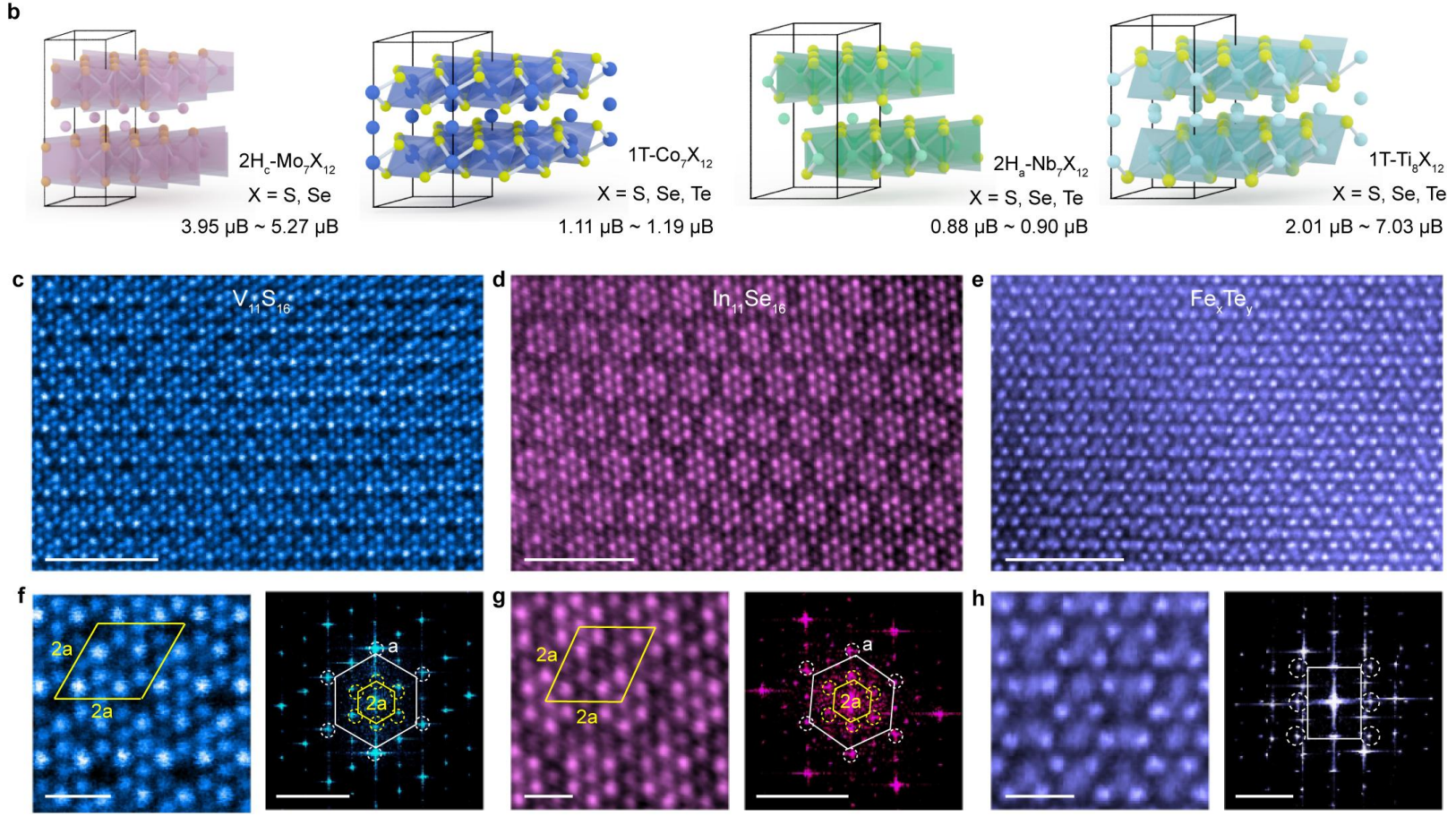

Figure 4. A library of ic-2d crystals. (a) Periodic table showing metal (highlighted blue) and chalcogen

399 (highlighted red) combinations that form ic-2d crystals according to our DFT calculation; the list is non-

400 exhaustive. Blue triangles indicate that the self-intercalation can be experimentally realized, whereas gray

401 triangles indicate that intercalation was not successful under our experimental conditions. Intrinsic

402 ferromagnetic $M X_{2}$ are highlighted by orange triangles. (b) DFT calculated ic- $2 d$ atomic models showing

403 ferromagnetism. STEM-ADF images of self-intercalated (c) $V_{11} S_{16}$, (d) $\ln _{11} \mathrm{Se}_{16}$, and (e) $\mathrm{Fe}_{\mathrm{x}} \mathrm{Te}_{\mathrm{y}}$. Their 
enlarged images and corresponding FFT patterns are depicted in (f-h), respectively. $2 \mathrm{~nm}$ in (c-e); $0.5 \mathrm{~nm}$

405 in (f-h); $5 \mathrm{~nm}^{-1}$ for all FFT patterns in (f-h).

406

407

408

409

410

411

412

413

414

415

416

417

418

419

420

421

422

423

424

425

\section{Methods}

MBE growth of self-intercalated TMD films. Ta-intercalated $T a_{x} S_{y}$ films were grown in a dedicated MBE chamber (base pressure $<6 \times 10^{-10}$ torr). Prior to the growth, the 2 -inch $\mathrm{SiO}_{2}$ substrates were degassed in the same chamber at $500{ }^{\circ} \mathrm{C}$ for $2 \mathrm{~h}$. Ultrapure Ta (99.995\%, Goodfellow) and S powders ( $99.5 \%$ Alfa Aesar) were evaporated from a mini electron-beam evaporator and a standard sulphur valved cracker, respectively. The flux density of Ta was precisely controlled by adjusting the flux current. The S cracker cell temperature was maintained at $110{ }^{\circ} \mathrm{C}$, and the flux density was controlled by the shutter of the cracker valve. The substrate temperature was maintained at 600 to $650{ }^{\circ} \mathrm{C}$ and the growth time is about 3 hours for all thin films. 25\% Ta-intercalated $\mathrm{Ta}_{9} \mathrm{~S}_{16}, 33.3 \% \mathrm{Ta}_{7} \mathrm{~S}_{12}$, and $50 \% \mathrm{Ta}_{10} \mathrm{~S}_{16}$ films were controlled grown when the Ta/S ratio is set as $\sim 1: 8, \sim 1: 6$, and $\sim 1: 5$, respectively. A slightly higher growth temperature will facilitate the self-intercalation process. After growth, both sources were turned off and the sample was further annealed for another 30 mins before cooling down to the room temperature. In-intercalated In $\boldsymbol{n}_{\boldsymbol{x}} \boldsymbol{S} \boldsymbol{e}_{y}$ were grown in a customized MBE chamber (base pressure $<6 \times 10^{-10}$ torr). Prior to growth, the $1 \mathrm{~cm}$ $\times 1 \mathrm{~cm} \mathrm{SiO}{ }_{2}$ substrate was degassed in the chamber at $600{ }^{\circ} \mathrm{C}$ for $1 \mathrm{~h}$. Ultrapure $\ln _{2} \mathrm{Se}_{3}$ powder (99.99\%) and Se pellets (99.999\%) were evaporated from a mini electron-beam evaporator and an effusion cell, respectively. The Se effusion-cell temperature was set at $150{ }^{\circ} \mathrm{C}$ with a hot-lip at $220^{\circ} \mathrm{C}$. The substrate temperature was maintained at $400^{\circ} \mathrm{C}$ and the growth time is about 2 hours. $\ln _{11} \mathrm{Se}_{16}$ films were controlled grown when the $\ln _{2} \mathrm{Se}_{3} / \mathrm{Se}$ ratio is set as $\sim 1: 3$.

CVD growth of self-intercalated TMD films. Ta-intercalated $T a_{x} S e_{y}$ crystals were grown by CVD. Prior to the growth, the $\mathrm{SiO}_{2}$ substrate was sequentially cleaned by water and acetone, followed by 5 min of $\mathrm{O}_{2}$ 
plasma. The furnace was purged by $300 \mathrm{sccm}$ Ar gas for 5 mins. Se powders and mixed $\mathrm{Ta} / \mathrm{TaCl}_{5}$ powders

427 were applied as precursors that were located upstream in a one-inch quartz tube. $40 \mathrm{sccm}$ Ar and $10 \mathrm{sccm}$

$428 \mathrm{H}_{2}$ were used as a carrier gas. The samples were grown at $800{ }^{\circ} \mathrm{C}$ for 30 mins. After growth, the sample was cooled down quickly in a continuous stream of Ar. $66.7 \%$ Ta-intercalated $\mathrm{Ta}_{8} \mathrm{Se}_{12}$, and $100 \%$ Ta430 intercalated $\mathrm{Ta}_{9} \mathrm{Se}_{12}$ were controlled grown when the Se powders and mixed $\mathrm{Ta} / \mathrm{TaCl}$ powders are $1 \mathrm{~g} / 15$ $\mathrm{mg} / 1.5 \mathrm{mg}$, and $1 \mathrm{~g} / 30 \mathrm{mg} / 3 \mathrm{mg}$, respectively. $\boldsymbol{V}$-intercalated $\boldsymbol{V}_{\boldsymbol{x}} \boldsymbol{S}_{\boldsymbol{y}}$ crystals were grown by CVD. Prior to

432 the growth, the $\mathrm{SiO}_{2}$ substrates were treated by the same method as indicated in growing $\mathrm{Ta}_{x} \mathrm{Se}_{\mathrm{y}}$. Two 433 quartz boats containing $0.5 \mathrm{~g} \mathrm{~S}$, and $0.3 \mathrm{~g} \mathrm{VCl}_{3}$ were loaded in the upstream of the one-inch quartz tube 434 serving as precursors. Carrier gases were $40 \mathrm{sccm}$ Ar together with $10 \mathrm{sccm} \mathrm{H}$. The sample was grown at $680{ }^{\circ} \mathrm{C}$ for 30 mins. After growth, the sample was cooled down quickly under the protection of $100 \mathrm{sccm}$ 436 Ar. Fe-intercalated $\mathbf{F e}_{x} \mathrm{Te}_{y}$ crystals were grown by CVD. Prior to the growth, the $\mathrm{SiO}_{2}$ substrates were 437 treated by the same method as indicated in growing $\mathrm{Ta}_{x} \mathrm{Se}_{\mathrm{y}}$. Two quartz boats containing Te (>99.997\%) 438 and $\mathrm{FeCl}_{2}(>99.9 \%)$ were placed in the upstream in sequence of the one-inch quartz tube serving as 439 precursors. The sample was grown at $600{ }^{\circ} \mathrm{C}$ for 30 mins. After growth, the sample was cooled down 440 quickly under the protection of $100 \mathrm{sccm}$ Ar.

441 Sample characterization. XPS was performed using SPECS XR 50 X-ray AI Ka (1486.6eV) source with a pass 442 energy of $30 \mathrm{eV}$. The chamber base pressure is lower than $8 \times 10^{-10} \mathrm{mbar}$. Raman spectra were collected 443 at room temperature using the confocal WiTec Alpha 300R Raman Microscope (laser excitation: $532 \mathrm{~nm}$ ).

444 STEM sample preparation, image characterization, and image simulation. The as-grown TMD films were 445 transferred via a PMMA method under the protection of graphene. Continuous graphene film was coated 446 on fresh $\mathrm{Ta}_{7} \mathrm{~S}_{12}$ film to protect the surface oxidation via conventional PMMA method. Subsequently, 447 graphene/ $\mathrm{Ta}_{7} \mathrm{~S}_{12}$ composites were immersed in $1 \mathrm{M} \mathrm{KOH}$ solution to detach the PMMA/ Ta7 $\mathrm{S}_{12}$ composite 448 from the $\mathrm{SiO}_{2}$ substrate, followed by rinsing in DI water. The PMMA/graphene/Ta7 $\mathrm{S}_{12}$ film was then placed 
onto $\mathrm{Cu}$ quantifoil TEM grid which was precoated with continuous graphene film ${ }^{44}$. The TEM grid was then

450 immersed in acetone to remove the PMMA films. Atomic-resolution STEM-ADF imaging was performed

451 on an aberration-corrected ARM200F, equipped with a cold field-emission gun and an ASCOR corrector

452 operating at $60 \mathrm{kV}$. The STEM-ADF images were collected using a half-angle range from $~ 81$ to $280 \mathrm{mrad}$

453 unless indicated elsewhere. The convergence semiangle of the probe was $\sim 30$ mrad. Image simulations

454 were done with the QSTEM package assuming an aberration-free probe with a probe size of $\approx 1 \AA$. The

455 convergence semiangle of the probe was set as $\sim 30 \mathrm{mrad}$, and the accelerating voltage is $60 \mathrm{kV}$ in line

456 with the experiments. The collection angle for HAADF and MAADF images were from 81 to $280 \mathrm{mrad}$ and

45730 to $110 \mathrm{mrad}$, respectively. The thermal diffuse scattering (TDS) was set as 30 with defocus value 0 . The

458 STEM-EDS were collected and processed in an Oxford Aztec EDS system

459 Device fabrication and measurements. MBE-grown $\mathrm{Ta}_{7} \mathrm{~S}_{12}$ and $\mathrm{CVD}$-grown $\mathrm{Ta}_{8} \mathrm{Se}_{12}$ were selected to 460 fabricate Hall-bar devices using e-beam lithography and e-beam evaporation of Ti/Au (2/60 nm). The MBE-

461 grown $\mathrm{Ta}_{7} \mathrm{~S}_{12}$ film was then etched into Hall-bar geometry using deep reactive ion etching. The final

462 devices were encapsulated with $h$-BN flakes using a dry-transfer method in the glovebox (both $\mathrm{O}_{2}$ and $\mathrm{H}_{2} \mathrm{O}$

463 less than $1 \mathrm{ppm}$ ), to avoid degradation of $\mathrm{Ta}_{7} \mathrm{~S}_{12}$ and $\mathrm{Ta}_{8} \mathrm{Se}_{12}$ under ambient conditions. Low-temperature

464 Transport measurements were carried out in Oxford Teslatron system. All resistances were derived from

465 four-terminal measurements using SR830 lock-in amplifier, with constant excitation current of $1 \mu \mathrm{A}$.

466 DFT calculations. First-principles calculations based on Density Functional Theory (DFT) were 467 implemented in the plane wave code VASP using the projector-augmented wave (PAW) potential 468 approach. For the exchange and correlation functional, both the local density approximation (LDA) and 469 the PBE flavor of the generalized gradient approximation are used and found no significant differences in 470 the results. A kinetic energy cutoff of $500 \mathrm{eV}$ is used for the $\mathrm{TaS}_{2}$. A Monkhorst Pack k-grid samplings with 471 a k-point density of $6.0 \AA^{-1}$ were used for geometry optimization. For thin-film calculations, a vacuum 
472 thickness of $20 \AA$ is added in the slab to minimize the interaction between adjacent image cells. Geometry

473 optimization was performed with the maximum force convergence criterion of $0.005 \mathrm{eV} / \AA$. To treat the

474 strong on-site Coulomb interaction of localized Ta-d orbitals, we use Dudarev's approach with an effective

$475 U$ parameter of $U_{\text {eff }}=3.0 \mathrm{eV}$. The zone center phonon modes were calculated by using the density

476 functional perturbation theory (DFPT) with the local density approximation functionals. High-throughput

477 DFT calculations were carried out with the electronic structure code GPAW ${ }^{45}$ following a semi-automated

478 workflow for maximal consistency and accuracy ${ }^{42}$. The relaxations of the self-intercalated bilayers are

479 done on a Monkhorst-Pack ${ }^{46}$ grid with a k-point density of $6.0 \AA^{-1}$ using the $\mathrm{PBE}^{47}$ and BEEF-vdW

480 functionals ${ }^{48}$ for describing exchange-correlation effects. $15 \AA$ vacuum is used in the out-of-plane direction

481 to avoid non-physical periodic interactions. The plane-wave expansion is cut-off at $800 \mathrm{eV}$. All systems are

482 relaxed until the maximum force on any atom is $0.01 \mathrm{eV} / \AA$ and the maximum stress on the unit cell is

$4830.002 \mathrm{eV} / \AA^{-3}$. All systems are calculated in the intercalated structure with both a spin-paired calculation

484 and a spin-polarized calculation. If the total energy of the spin-polarized structure is found to be over 0.01

$485 \mathrm{eV} /$ atom lower than the spin-paired structure, the structure is concluded to be magnetically more stable

486 than its non-magnetic counterpart. The atomic structures of calculated self-intercalated TMDs ( $33.3 \%$ and

$48766.7 \%$ intercalation concentration) were presented in Supplementary Fig. 29, in which the polymorphism

488 of single layer $\mathrm{MoX}_{2}, \mathrm{WX}_{2}, \mathrm{NbX}_{2}$, and $\mathrm{TaX}_{2}(\mathrm{X}=\mathrm{S}$, Se, and Te) reveals an $\mathrm{H}$-phase, whereas the rest TMDs

489 are T-phase taking an $A A$ stacking polytype. $\mathrm{MoX}_{2}$, and $\mathrm{WX}_{2}$ comply the $A A^{\prime}$ stacking order whereas $\mathrm{NbX}_{2}$,

490 and $\mathrm{TaX}_{2}$ take the $A B^{\prime}$ stacking registry. All intercalants occupy the octahedral vacancies in the vdW gap.

491 Method references:

492 42. Haastrup, S. et al. The Computational 2D Materials Database: High-throughput modeling and

493 discovery of atomically thin crystals. 2D Materials 5, 042002 (2018).

494 44. Wang, H. et al. High-quality monolayer superconductor NbSe2 grown by chemical vapour

495 deposition. Nat. Commun. 8, 394 (2017). 
45. Enkovaara, J. et al. Electronic structure calculations with GPAW: A real-space implementation of the projector augmented-wave method. Journal of Physics Condensed Matter 22, 253202 (2010).

46. Monkhorst, H. J. \& Pack, J. D. Special points for Brillouin-zone integrations. Phys. Rev. B 13, 51885192 (1976).

47. Perdew, J. P., Burke, K. \& Ernzerhof, M. Generalized gradient approximation made simple. Phys. Rev. Lett. 77, 3865-3868 (1996).

502

48. Wellendorff, J. et al. Density functionals for surface science: Exchange-correlation model

503 development with Bayesian error estimation. Phys. Rev. B 85, 235149 (2012).

504 Data availability. The main data supporting the findings of this study are available within the paper and 505 its Supplementary Information. Extra data are available from the corresponding authors upon request. 